\title{
An Analysis of Synergies under Strategic Mergers and Acquisitions
}

\author{
Wang Yihui \\ Major of Finance, Business School, University of Iowa, 52242
}

Keywords: Strategy; Mergers and Acquisitions; Synergies

\begin{abstract}
With the continuous transformation from the planned economy to the market economy, the number of reorganizations and acquisitions between enterprises in China has increased, and the mergers and acquisition are dominant. As the market economy continues to deepen, the mergers and acquisitions of enterprises have undergone major changes. The mergers and acquisitions among companies have evolved from simple form to the strategic pattern of combing industrial integration and expansion. This article combines the relevant information of strategic mergers and acquisitions, to conduct a comprehensive and hierarchical analysis on the synergies produced by companies due to mergers and acquisitions, and study the risks associated with the synergies of mergers and acquisitions. Based on the analysis results, the solutions to reduce relevant risks are proposed, which are expected to provide reference for future enterprise mergers and acquisitions.
\end{abstract}

\section{Introduction}

With the continuous development of the market economy, there have been more and more Chinese companies undergoing the corporate restructuring and enterprise merger and acquisition, and the traditional enterprises mergers and acquisitions have changed greatly. In order to ensure the healthy and sustainable development themselves, many enterprises becomes cautious toward mergers and acquisitions, and the mergers and acquisitions between companies have transitioned from simple form to strategic pattern. The aim of strategic mergers and acquisitions is to achieve the ultimate goal of synergy effect. The synergy effect refers to the phenomenon of "one plus one is greater than two" in simple terms, which means that the overall benefit generated by the merged company is greater than the total of the benefits produced by previous separate companies, and it is an important indictor to judge whether the merge is successful. In this paper, a representative enterprise in the Internet industry of Alibaba is selected as a study case, to propose further suggestions and prospects for Chinese enterprises' strategic mergers and acquisitions through analysis, which is also expected to provide theoretical basis for mergers and acquisitions of other enterprises in China.

As China's economic globalization progresses and the market economy continues to accelerate, all walks of life in China have ushered in a golden period of development [1]. With the development of the times and the arrival of the media era, the development of Internet companies has been promoted further, which has shortened the influence factors of regional distance to a certain extent, and has narrowed the distance between people as well as has a very important influence on the details and quality of daily life [2]. Regarding the overall industry development trend of Internet companies, it is expected that the number of Internet users in China will reach 890 million in 2018.

According to the statistical data of relevant departments, the economic aggregate of China's Internet market has reached 6 trillion $¥$ in 2017, of which amplification is up to 29.6\%. It is expected that there is new breakthrough in the China's Internet scale in 2018, of which amplification will be up to $26.4 \%$, and its economic aggregate will reach 7 trillion and 654 billion $¥$ (as shown in Fig.2). 


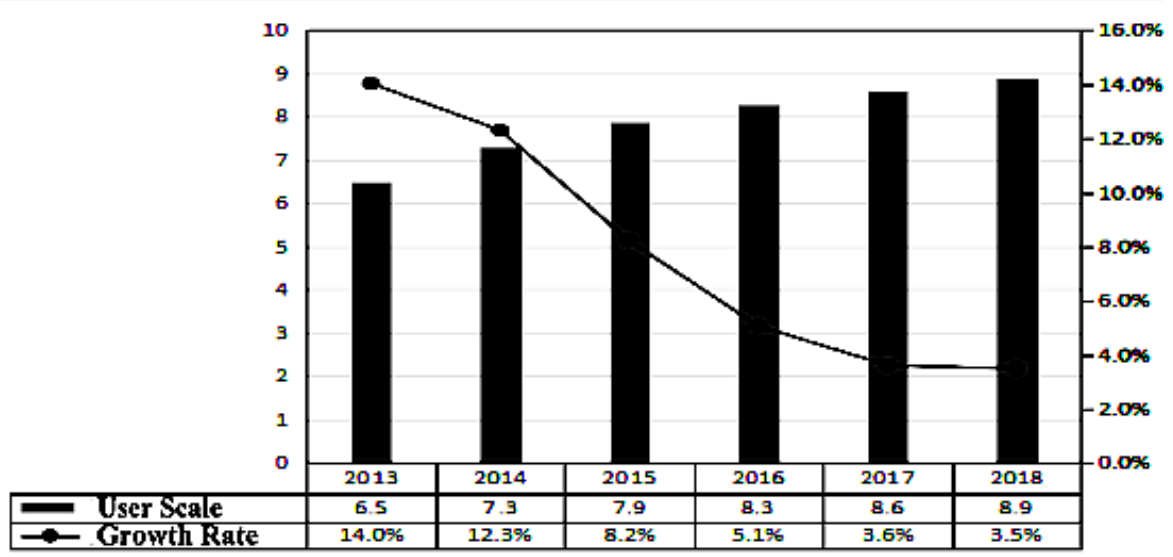

Fig.1 The Growth Situation of Internet Users in China

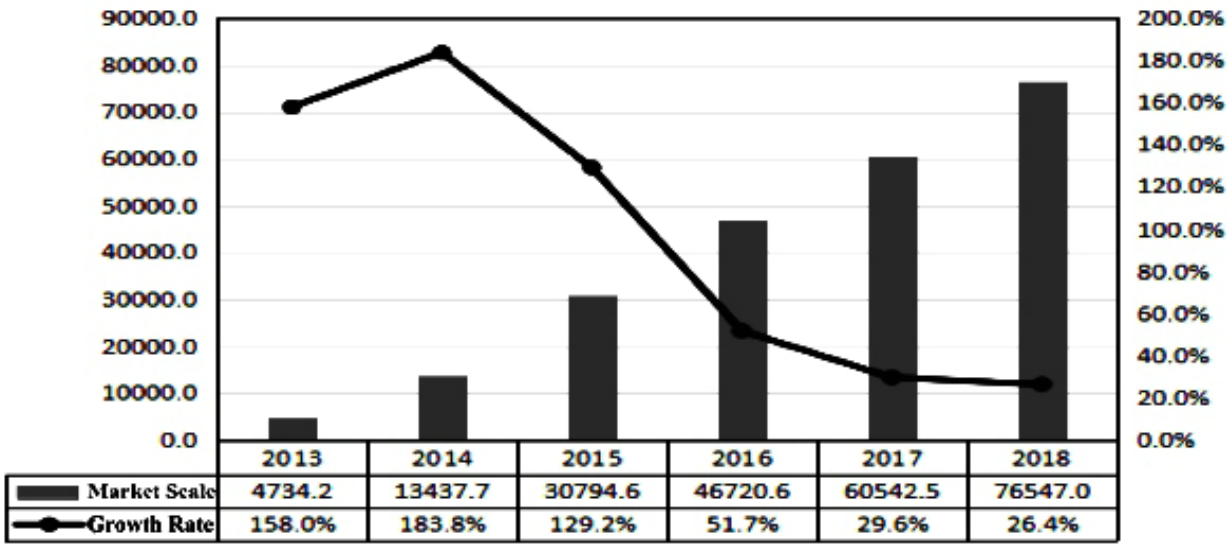

Fig.2 The Growth Situation of Internet Market Scale in China

With the rapid development of the economy, the China's merges and acquisitions market has been booming. The enterprise merges and acquisitions is very normal under the background of market economy. Throughout the development history of mergers and acquisitions, there has been a total of five mergers and acquisitions upsurges in the world, where the "enterprise remolding" was achieved.

Tab.1 Mergers and Acquisitions Upsurges History of Each Industry in the World

\begin{tabular}{|c|c|c|c|c|c|}
\hline Project & $\begin{array}{c}\text { Number of } \\
\text { Cases }\end{array}$ & Proportion & $\begin{array}{l}\text { Amount of } \\
\text { Merger and } \\
\text { Acquisition } \\
\text { ( billion } ¥ \text { ) }\end{array}$ & Proportion & $\begin{array}{c}\text { Average of } \\
\text { Merger and } \\
\text { Acquisition } \\
\text { Amount } \\
\text { (billion } ¥ \text { ) }\end{array}$ \\
\hline Internet & 656656 & $24.4 \%$ & 2927.18 & $28.0 \%$ & 4.46 \\
\hline Finance & 220 & $8.2 \%$ & 1079.34 & $10.3 \%$ & 4.91 \\
\hline Medical Health & 239 & $8.9 \%$ & 665.97 & $6.4 \%$ & 2.79 \\
\hline $\begin{array}{l}\text { Machinery and } \\
\text { Equipment }\end{array}$ & 348 & $12.9 \%$ & 716.75 & $6.9 \%$ & 2.06 \\
\hline Real Estate & 148 & $5.5 \%$ & 1302.65 & $12.5 \%$ & 8.80 \\
\hline $\begin{array}{l}\text { Entertainment } \\
\text { Media }\end{array}$ & 109 & $4.0 \%$ & 880.01 & $8.4 \%$ & 8.07 \\
\hline $\begin{array}{l}\text { Communication } \\
\text { Engineering }\end{array}$ & 283 & $10.5 \%$ & 720.56 & $6.9 \%$ & 2.55 \\
\hline Motor Traffic & 134 & $5.0 \%$ & 663.39 & $6.4 \%$ & 4.95 \\
\hline Retail Industry & 115 & $4.3 \%$ & 432.94 & $4.1 \%$ & 3.76 \\
\hline $\begin{array}{l}\text { Energy and } \\
\text { Minerals }\end{array}$ & 261 & $9.7 \%$ & 761.02 & $7.3 \%$ & 2.92 \\
\hline The Other & 179 & $6.6 \%$ & 87.44 & $0.8 \%$ & 0.49 \\
\hline Total & 2, 692 & $100 \%$ & $10,437.25$ & $100 \%$ & 3.88 \\
\hline
\end{tabular}


According to the internet report published by Tsinghua Science and technology research center in 2017, in the past few years, the number of China's merge and acquisition market transactions has been increasing year by year, of which growth amplitude has been also increasing and will continue to increase in the future [3]. In this way, the merge and acquisition has become an effective means to conduct economic structure adjustment and optimize resource under the background of market economy (the concrete data is shown as the following table).

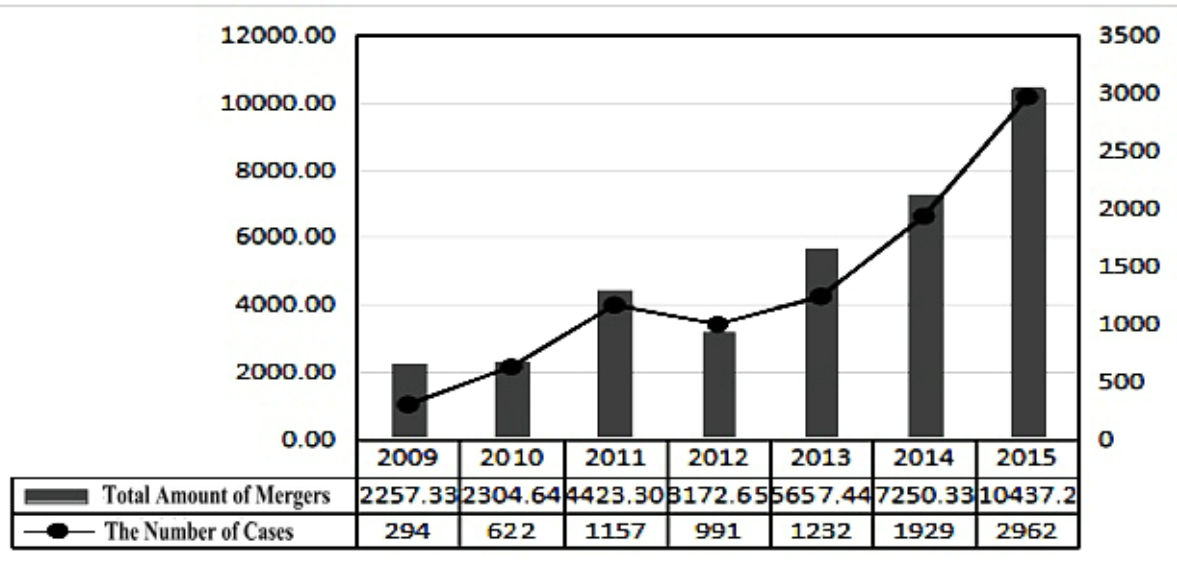

Fig.3 The Growth Situation of Merge and Acquisition Market Transactions in China

\section{The Relationship between Strategic Merger and Synergy}

Strategic mergers and acquisitions can be divided into three major categories based on industrial structure: horizontal, vertical and mixed mergers and acquisitions, and the synergies can also be divided into three categories in terms of sources: management, operations, and finance synergistic effects.

In terms of mergers and acquisitions effect, each category is different from each other. First of all, horizontal mergers and acquisitions should be combined with management synergies, which is based on the merger and acquisition of similar companies, to improve the current development status of enterprises, so as to achieve the ultimate goal of improving the company's benefits. The synergy effect of horizontal mergers and acquisitions should be mainly reflected in the management cost savings and the effective use of resources. Secondly, the vertical mergers and acquisitions should be combined with business synergy effects, and the relevant contact costs and transaction costs are expected to be minimized through mergers and acquisitions. Besides, the product transaction costs and marketing costs are expected to be reduced, and the ties of all segments are expected to be strengthen, to achieve a synergistic effect on operation [4]. Finally, the mixed mergers and acquisitions should be combined with financial synergies, and the financial benefits are expected to be increased through mergers and acquisitions of different types of enterprises, which is mainly reflected in the improvement of financial capabilities, effects and so on.

\subsection{The Strategic Mergers and Acquisitions of Alibaba}

As the Alibaba founder Ma Yun once said, if an enterprise is expected to be operated over one century, of which leaders must continue tap the market needs and open up new business directions, so as to take the constantly updating the development direction as the backbone of the company's future development [5]. The corporate expansion strategy can be divided into two categories of integration and diversification, and the former is equipped with its own special classification. Through sorting out the Alibaba's merge and acquisition activities in this study, it is found that a horizontal integration and vertical integration strategy is mainly adopted by Alibaba in the previous mergers and acquisitions period, while the diversification strategy is adopted in the later period (The main activities are shown in Tab.2).

Horizontal Integration Strategy. Horizontal integration strategy mainly refers to the process of production and sales are connected in series to form collective management, of which aim is to 
promote the effective use and concentration of resources essentially. In fact, Alibaba belongs to the e-commerce industry, which is relatively unified with Jingdong Mall, Jumei, and Mushroom Street. In July 2011 and April 2013, Alibaba merged Meituan Company and Kuaidi Dache Company respectively, which are the embodiment of their horizontal integration strategy.

Vertical Integration Strategy. Vertical integration refers to the business layout of multiple production processes along industrial chain, which can divided into the two categories of backward integration and forward integration [6]. Backward integration mainly refers to the upstream extension of the enterprise and the enterprise's self-supply of raw materials. The forward integration refers to the extension of the enterprise to the downstream, where the product deep-processing is achieved and the special channels to sell products are established. As an e-commerce platform, Alibaba has become a mid-stream point in distributing trading information and achieving trading, in which the upstream is the e-commerce service provider, and the downstream is the entity seller, payment platform and logistics company.

Diversification Strategy. Diversification strategy is a kind of expansion strategy, based on which one company can achieve the layout of other industries, such as Alibaba's expansion into financial, film, television and sports industry. In the initial expansion period, Alibaba's expansion is mainly around main business to achieve integrated expansion, but with the continuous development of the company, there has been a diversified expansion. Throughout the development strategies of large companies at home and abroad, the principal route is from single strategy to diversified strategy, to achieve the goal of reducing business risks and increasing profits, and ultimately achieving the long-term development of company.

Tab.2 Alibaba’s Merge and Acquisition Activity Summary

\begin{tabular}{|c|c|c|c|}
\hline $\begin{array}{l}\text { Merge and } \\
\text { Acquisition } \\
\text { Strategy }\end{array}$ & Type of Enterprise & & Acquired Enterprise \\
\hline $\begin{array}{c}\text { Horizontal } \\
\text { Integration Strategy }\end{array}$ & E-commerce Platform & & eituan, Yueke, Software \\
\hline \multirow{10}{*}{$\begin{array}{l}\text { Vertical Integration } \\
\text { Strategy }\end{array}$} & \multirow{7}{*}{ Upstream } & $\begin{array}{l}\text { E-commerce service } \\
\text { provider }\end{array}$ & $\begin{array}{l}\text { E-commerce supplier Lazada, CITIC 21st } \\
\text { Century, Meizu, Softbank Robot Holdings, } \\
\text { Suning Cloud Business }\end{array}$ \\
\hline & & Search Interfaces & Yahoo China \\
\hline & & $\begin{array}{l}\text { Entertainment } \\
\text { Entrance }\end{array}$ & Youku Tudou Inc, XiamiMusic \\
\hline & & Social Entrance & Sina Weibo, MOMO, Tango \\
\hline & & Life Entrance & 58 Home, Eleme, Koubei, DDMAP, qyer \\
\hline & & Browser Entrance & $\mathrm{UC}$ \\
\hline & & Download Entrance & Pea Pod \\
\hline & \multirow{3}{*}{ Downstream } & Logistics Company & Haier Rishun、 Singapore Post \\
\hline & & Entity Buyer & Yintai Business \\
\hline & & Offline Travel & $\begin{array}{l}\text { Amap, Kuaidi Dache, Didi Chuxing, Lyft } \\
\text { Car-Pooling }\end{array}$ \\
\hline \multirow{5}{*}{$\begin{array}{l}\text { Diversification } \\
\text { Strategy }\end{array}$} & \multicolumn{2}{|c|}{ Financial Industry } & $\begin{array}{c}\text { Tianhong Asset Management, Hundsun } \\
\text { Technologies }\end{array}$ \\
\hline & \multicolumn{2}{|c|}{ Entertainment Industry } & Huayi Brothers Media Group \\
\hline & \multicolumn{2}{|c|}{ Culture Industry } & $\begin{array}{c}\text { China's Cultural Media, Wasu Media, } \\
\text { AdChina, ENLIGHT MEDIA }\end{array}$ \\
\hline & \multicolumn{2}{|c|}{ Sports Industry } & Guangzhou Evergrande Football Club \\
\hline & \multicolumn{2}{|c|}{ News Industry } & $\begin{array}{c}\text { South China Morning Post, China Business } \\
\text { Network }\end{array}$ \\
\hline
\end{tabular}




\subsection{Synergy Effect under Financial Data}

The synergy effect refers to that various indicators of enterprise have been improved after merger, so as to achieve the ultimate goal of the enterprise merger and acquisition that realizing the development of the enterprise.

Alibaba implemented strategic merger and acquisition three years ago, of which business data in 2017 have not been released. Therefore, the average of the growth rate of operating income in past three years before the merger and acquisition is used, to predict the trend of operating income growth rate in the future, and the growth rate of operating income after the merger and acquisition is $68 \%$ and $39 \%$ respectively [7].

Tab.3 Increase Rate of Business Revenue

\begin{tabular}{cccc|cc}
\hline \hline \multirow{2}{*}{ Year } & \multicolumn{3}{c|}{ Before Acquisition } & \multicolumn{2}{c}{ After Acquisition } \\
\cline { 2 - 5 } Project & 2012 & 2013 & 2014 & 2015 & 2016 \\
\hline $\begin{array}{c}\text { Prime Operating } \\
\text { Revenue (million } ¥ \text { ) }\end{array}$ & 20,025 & 34,517 & 550,204 & 76,204 & 101,143 \\
Revenue Growth Rate & $80.21 \%$ & $72.37 \%$ & $52.11 \%$ & $45.14 \%$ & $37.23 \%$ \\
\hline \hline
\end{tabular}

When estimating the gross profit margin of Alibaba group, the gross profit margin before and after the method used was $71 \%$ and $67 \%$ respectively.

Tab.4 Analysis of Gross Margin

\begin{tabular}{cccc|cc}
\hline \hline \multirow{2}{*}{ Year } & \multicolumn{3}{c|}{ Before Acquisition } & \multicolumn{2}{c}{ After Acquisition } \\
\cline { 2 - 6 } Project & 2012 & 2013 & 2014 & 2015 & 2016 \\
\hline Operating Revenue & 20,025 & 34,517 & 52,504 & 76,204 & 101,143 \\
Operating Costs & 6554 & 9,719 & 13,369 & 23,834 & 34,355 \\
Gross Margin & 13,471 & 24,798 & 39,135 & 52,370 & 66,788 \\
Gross Margin Rate & $67.27 \%$ & $71.84 \%$ & $74.54 \%$ & $68.72 \%$ & $66.03 \%$ \\
\hline \hline
\end{tabular}

Based aforementioned displayed data, it shows that the internal value of Alibaba's has increased obviously after merger and acquisition, thus it can be concluded that Alibaba has realized the synergy effect through strategic merger and acquisition

\section{Synergy Effect Mechanism and Cost}

Based on the above displayed data of Alibaba enterprises, Alibaba has paid a considerable cost while acquiring benefits, and its action mechanism is an important guarantee to realize the synergy effect, which will be carefully analyzed as follows:

\section{1 .Mechanism of Action}

The concrete manifestation of synergy is core competitiveness, which is manifested in two aspects: concentration and diffusion. The core competitiveness enables enterprises to gain competitive advantages in their competitive activities and forms long-term competitive advantages. The generation of synergy requires four basic elements: firstly, enterprises must set clear goals before mergers and acquisitions; secondly, enterprises must have systematic integration capabilities so that the synergy effect can be truly realized; thirdly, enterprises should have the ability of multicultural management and integration, which can integrate the cultures of the two enterprises and create a common culture; finally, enterprises pay more attention to the market, technology, management and other aspects after the merger and acquisition, and implement strong management to achieve the goal of the merger and acquisition. 


\subsection{Realization Cost}

The synergy effect of strategic mergers and acquisitions requires enterprises to pay a certain cost in advance before bringing benefits to enterprises.

Premium. In the process of strategic mergers and acquisitions, the price of mergers and acquisitions is often higher than its actual value. Therefore, the emergence of market premium requires enterprises to collect information of target enterprises, and conduct in-depth research and analysis to determine the upper limit of merger and acquisition premium.

Integration Cost. The enterprise mergers and acquisitions cannot be separated from the cost consumed in the process of integration, and it is necessary for enterprise to regulate and control the operating procedures, technological process and process personnel of the merged enterprises again, so as to provide the basic guarantee for maximizing the benefits.

Upfront Expense. During the transaction process of strategic merger and acquisition, enterprises need to pay for the information collection and other expenses in the early stage, and only when the early stage foundation is laid, the realization of synergy effect can be guaranteed.

\section{Synergy Effect Sources and Risks}

\subsection{Synergy Sources}

The generation of synergistic effect mainly comes from three major directions: saving capital expenditure, reducing cost and increasing income, which can be helpful for enterprises to realize the promote of their interests and finally achieve the purpose of synergy.

\subsection{Realization Risk}

The realization risk of synergy effect represents general problems that appear in adding enterprise value. The cost of the merger and acquisition strategy is certain, but the synergy effect is extremely unstable, which can have a great impact on enterprise. The realization risks can be divided into internal risks and external risks.

Internal Risk. First of all, the consequence of merge and acquisition transaction has a very important influence on the realization of synergy and the integration of resources. If there are some problems in the process of integration, it will affect the realization of synergy effect, increase various costs and reduce the benefits of enterprises.

External Risk. Specifying the corresponding development strategy is important for realizing the synergy effect, and the environmental factors must be considered fully in the process of making plan, because it will greatly increase the frequency of risk occurrence once environmental factors change.

\subsection{Risk Control}

In fact, the synergy risk exists indeed. Under the condition that the external conditions of mergers and acquisitions are certain, it is very difficult to control the environmental risks, which is usually controlled by choosing external environment to reduce risk frequency. In order to avoid the internal risks, the risk measures are mainly used to increase the impact on mergers and acquisitions and to control the risks vigorously.

Reasonable Assessment. First of all, before the merger and acquisition, the enterprise managers should conduct a detailed investigation of all aspects of the targeted enterprises and predict its future profitability reasonably, to develop proper pricing strategy of merger and acquisition on the basis of reasonable evaluation, which can reduce the evaluation risk.

Adhere to Sustainable Core Competitive Advantage. Before mergers and acquisitions, it is necessary to understand opponents fully, analyze the actual situation of enterprises, and formulate reasonable strategies for mergers and acquisitions that can constantly change with the everchanging actual situation. In addition, it is necessary to determine whether mergers and acquisitions can achieve sustainable core competitive advantages so as to be more competitive in the competition of the entire industry. 
Carrying Out Proper and Effective Merger and Acquisition Integration. The integration plan includes industry, management and cultural integration. In the process of integration, it is necessary to adhere to the leading industries of enterprises, maintain technological advantages, and continuously introduce new and high technologies. In addition, the advanced management mode of merge and acquisition enterprises is expected to be integrated with the internal management mechanism of the target enterprises, to reduce costs, improve efficiency, and formulate a mature corporate culture finally.

Reducing the Risk of Principal-Agent Relationship. The asymmetry of information between managers and shareholders leads to the risk of choice and morality in the market. As a result, the methods of supervision and encouragement needed to be adopted to reduce the risks and maximize the benefits to the enterprise generated by the synergy effect.

\section{Conclusion}

At the beginning of this study, taking the Alibaba group as an example, the current merger and acquisition status of internet enterprises is analyzed. Through the enumeration of data, the synergy effect of Alibaba group after the strategic merger and acquisition that can promote its internal value is illustrated. At the end of the article, the current synergy effect and related issues of strategic mergers and acquisitions as well as relevant opinions are expounded, expected to improve the theoretical basis for future strategic mergers and acquisitions.

\section{References}

[1] Loukianova A, Nikulin E, Vedernikov A. Valuing synergies in strategic mergers and acquisitions using the real options approach[J]. 2017.

[2] Bena J, Li K. Corporate innovations and mergers and acquisitions[J]. The Journal of Finance, 2014, 69(5): 1923-1960.

[3] Zheng N, Wei Y, Zhang Y, et al. In search of strategic assets through cross-border merger and acquisitions: Evidence from Chinese multinational enterprises in developed economies[J]. International Business Review, 2016, 25(1): 177-186.

[4] Ferreira M P, Santos J C, de Almeida M I R, et al. Mergers \& acquisitions research: A bibliometric study of top strategy and international business journals, 1980-2010[J]. Journal of Business Research, 2014, 67(12): 2550-2558.

[5] Pathak H P. Motives for Mergers and Acquisitions in the Nepalese Perspective[J]. Economic Literature, 2016, 13: 9-18.

[6] Zheng N, Wei Y, Zhang Y, et al. In search of strategic assets through cross-border merger and acquisitions: Evidence from Chinese multinational enterprises in developed economies[J]. International Business Review, 2016, 25(1): 177-186.

[7] Dutordoir M, Roosenboom P, Vasconcelos $M$. Synergy disclosures in mergers and acquisitions[J]. International Review of Financial Analysis, 2014, 31: 88-100. 